ORIGINAL ARTICLE

\title{
Incidence of serious injury and death during sport and recreation activities in Victoria, Australia
}

\author{
B J Gabbe, C F Finch, P A Cameron, O D Williamson
}

Br J Sports Med 2005;39:573-577. doi: 10.1136/bjsm.2004.015750

See end of article for authors' affiliations

.........................

Correspondence to: Dr Gabbe, Monash University, Epidemiology and Preventive Medicine, Central and Eastern Clinical School, Alfred Hospital, Commercial Road, Melbourne 3004, Victoria, Australia; belinda.gabbe@med. monash.edu.au

Accepted

21 December 2004

\begin{abstract}
Background: Participation in sport and recreation is widely encouraged for general good health and the prevention of some non-communicable diseases. However, injury is a significant barrier to participation, and safety concerns are a factor in the decision to participate. An understanding of the sport/recreation activities associated with serious injury is useful for informing physical activity choices and for setting priorities for the targeting of injury prevention efforts.

Objectives: To describe the epidemiology of serious injuries sustained in sport/recreation activities by adults in Victoria, Australia.

Methods: The Victorian State Trauma Registry and the National Coroner's Information Service were used to identify and describe sport/recreation related serious injuries, including deaths, occurring during the period July 2001 to June 2003. Age adjusted rates of serious injury and death were calculated using participation figures for each sport and general population data.

Results: There were 150 cases of serious injury and 48 deaths. The rates of serious injury and death were 1.8 and 0.6 per 100000 participants per year respectively. Motor, power boat, and equestrian sports had the highest rates of serious injury. Most deaths were due to drowning.

Conclusion: Although the risk of serious injury through sport/recreation participation is low, motor, power boat, and equestrian sports should be priorities for further research into injury prevention. Most sport/ recreation related deaths are due to drowning, highlighting this area for prevention efforts.
\end{abstract}

$\mathrm{E}$ ncouraging participation in sport and recreation has become a major public health priority internationally because of rising rates of obesity and the potential for an increase in diseases related to a sedentary lifestyle. However, participation is not without risk, and injuries can occur. The cost of sport/recreation related injuries in Australia was recently estimated at $\$ 1.8$ billion per annum. ${ }^{1}$ Injury has also been identified as a significant barrier to participation in sport/recreation, and safety concerns are a factor in the decision to participate. ${ }^{2}$

Published studies have described emergency department presentations for sport/recreation injury ${ }^{3-6}$ or serious injuries related to individual sports. ${ }^{7-10}$ Previous population based studies have been limited because they did not differentiate between sports, ${ }^{6}$ they used population rather than participation figures to calculate injury rates, ${ }^{6}{ }^{11}$ or they excluded fatalities. ${ }^{12}$ This information is necessary to identify the sport/ recreation activities that result in serious injury, to highlight potential areas for prevention, and to set priorities for injury prevention research.

This study aimed to describe the epidemiology of serious sport/recreation injuries sustained by adults across the total population in Victoria, Australia over a two year period.

\section{METHODS}

\section{Setting}

The state of Victoria has a population of 4.7 million people, accounting for $24 \%$ of all Australians. The majority (73\%) of Victorians live in metropolitan Melbourne, and population surveys suggest that just over 3.0 million (or $78.5 \%$ ) adult Victorians participate in sport/recreation activities. ${ }^{13}$

\section{Databases}

Patients, aged 15 years and over, with sport/recreation related injury, who presented to hospital for treatment or who died before reaching hospital were identified through the
Victorian State Trauma Registry (VSTR) and the National Coroner's Information System (NCIS).

The VSTR is a state wide trauma registry which captures information about all major trauma patients in Victoria. ${ }^{14}$ Major trauma, as defined by the VSTR, includes death, admission to an intensive care unit, an injury severity score (ISS) $>15$, and urgent surgery (within 24 hours of admission and surgery involving intracranial, intrathoracic, intraabdominal injury or fixation of spinal or pelvic fractures). ${ }^{14}$

The VSTR records patient and injury details. Diagnoses are coded according to the abbreviated injury scale (AIS), ${ }^{15}$ and the ISS is calculated from the AIS to give an overall rating of the severity of the patient's injuries. ${ }^{16}$ The VSTR also collects information about outcomes, including mortality, length of hospital stay, and the destination after discharge from an acute hospital. It is estimated that more than $90 \%$ of all major trauma cases in Victoria are registered on the VSTR.

Cases meeting any of the following criteria that occurred between July 2001 and June 2003 (inclusive) were extracted for analysis:

- Activity at the time of injury, coded as "sports and active recreation" or "leisure"

- Place of injury, coded as "athletic or sports area" or "place for recreation"

- Cause of injury, coded as "motorcycle driver or passenger", "pedal cyclist" or "horse related"

Cases involving motorcycle and pedal cyclists that occurred on a road, street, or highway were excluded as it was not possible to determine whether they occurred as a result of

Abbreviations: AIS, abbreviated injury scale; ERASS, Exercise, Recreation and Sport Survey; ISS, injury severity score; NCCSIR, National Centre for Catastrophic Sports Injury Research; NCIS, National Coroner's Information System; VSTR, Victorian State Trauma Registry 
recreational or transport activities. All cases involving offroad (trail) motorcycles, motorcycle racing, BMX riding, and mountain bikes were included. The sport/recreation activity participated in at the time of injury was coded from the detailed text narrative of the injury event. The VSTR inclusion criteria exclude many near drowning cases, but cases meeting the stated criteria were included in this study.

The NCIS is a national centralised data storage and retrieval system for coronial information. The activity at the time of injury was used to identify sport/recreation related deaths for the period July 2001 to June 2003. Cases coded as participating in "informal sport and active recreation", "organised sport and active recreation", and "leisure activity" were extracted for analysis. The use of identifiable data from the VSTR and the NCIS prevented double counting of cases across the datasets.

Ethics approval was obtained from all participating hospitals and the Monash Standing Committee on Ethics in Research involving Humans. These ethics approvals included access to the NCIS.

\section{Data management and analysis}

Descriptive statistics were used to define the profile of participants who were seriously injured. As participants could sustain more than one injury, the body region injured and nature of injuries sustained were expressed as the proportion of all injuries.

Crude serious injury rates were calculated by dividing the mean annual number of deaths or serious injuries over the two year period by the number of persons participating in sport or physical activity as determined by the Exercise, Recreation and Sport Survey (ERASS). ${ }^{17}$ Age and sex specific rates were estimated by direct standardisation using age and sex specific population data from the 2001 Australian Census. ${ }^{18}$

The rate of serious injury per 100000 participants per year for each activity was calculated by dividing the number of serious injuries sustained each year by the number of persons participating in that particular sport in Victoria. ${ }^{17}$ If Victorian participation figures were not directly available, they were derived from national figures because there was no reason to believe that participation in Victoria would differ from that across Australia for the sports where state specific figures were not available.

Table 1 Sport and recreational activities resulting in serious injury and death in Victoria, Australia 1 July 2001 to 30 June 2003 (inclusive) $(n=198)$

\begin{tabular}{llll}
\hline Sport or recreation activity & No & $\%$ of cases & $\begin{array}{l}\text { Rate of injury } \\
\text { and death }\end{array}$ \\
\hline Motor sports & 63 & 31.8 & 113.3 \\
Horse riding & 28 & 14.1 & 29.7 \\
Australian football & 17 & 8.6 & 5.5 \\
Water skiing/power boating & 17 & 8.6 & 31.5 \\
Swimming & 14 & 7.1 & 1.8 \\
Aero sports & 12 & 6.1 & $\mathrm{n} / \mathrm{a}^{*}$ \\
Fishing & 10 & 5.1 & 9.2 \\
BMX and mountain biking & 5 & 2.5 & $\mathrm{n} / \mathrm{a} \dagger$ \\
Snow skiing & 4 & 2.0 & 2.5 \\
Rock climbing & 4 & 2.0 & 6.6 \\
Other & 24 & 12.1 & $\mathrm{n} / \mathrm{a}$ \\
Total & 198 & 100.0 & - \\
\hline
\end{tabular}

The rate of injury and death is given per 100000 participants per year. *Participation rates not available for Victoria or Australia. †Participation rates not specific to BMX and mountain biking $\mathrm{n} / \mathrm{a}$, Not available.
Table 2 Rate of sport and recreation related serious injury and death in Victoria, Australia 1 July 2001 to 30 June 2003 (inclusive)

\begin{tabular}{lll}
\hline Sex & $\begin{array}{l}\text { Rate of } \\
\text { serious injury }\end{array}$ & Death rate \\
\hline Male & & \\
$\quad$ Crude & & 1.5 \\
$\quad$ Age adjusted & 3.0 & 1.2 \\
Female & 0.8 & 0.1 \\
$\quad$ Crude & 0.6 & 0.1 \\
$\quad \begin{array}{l}\text { Age adjusted } \\
\text { Combined } \\
\quad \text { Crude }\end{array}$ & 2.5 & 0.8 \\
$\quad$ Age adjusted & 1.8 & 0.6 \\
\hline $\begin{array}{l}\text { The rates of serious injury and death are given per } 100000 \text { participants } \\
\text { per year. }\end{array}$ &
\end{tabular}

\section{RESULTS}

\section{Sport and recreation related serious injury and death} (2001-2003)

Overall, there were 150 reported cases of serious injury and 48 deaths related to sport/recreation participation between 1 July 2001 and 30 June 2003 (inclusive). In eight cases, the injured person died during their acute hospital stay and the remainder died at the scene and were investigated by the state coroner. Of the deaths, $69 \%$ were due to drowning. In most of the cases, the injured person was male $(81 \%)$ and less than 45 years of age $(76 \%)$. In most cases $(82 \%)$, the ISS was above 15. Table 1 shows the profile of the sport/recreation activities resulting in serious injury and death.

Motor sports accounted for $32 \%$ of all cases, with most $(\mathrm{n}=52)$ related to motorbike riding. Over half $(54 \%)$ of the motorbike riding related cases were due to a loss of control and fall from the bike, and 19\% occurred when a trail bike struck a stationary object such as a tree. There were five deaths among the motor sport cases, three due to motorbike riding and one each due to car racing and all terrain vehicle (quad bike) riding.

Cases were also common in horse riding $(\mathrm{n}=28)$ and Australian football $(\mathrm{n}=17)$, but no deaths were reported. Most of the horse riding cases ( $82 \%$ ) were due to a fall from a horse. All those in Australian football were the result of being struck by or colliding with another player-for example, during a tackle. Seventeen cases were the result of participation in water skiing or power boating. Ten were fatalities, with nine of these deaths due to drowning. Twelve cases were related to participation in aero sports, and half of these were fatalities. The types of aero sport resulting in serious injury and death were hang gliding, parachuting, paragliding, and piloting a glider, and all injuries/deaths were due to an awkward landing or crash. All fishing related cases were fatalities due to drowning. The "other" group of sports included rollerblading, skate boarding, basketball, cricket, netball, boxing, surfing, bushwalking, rugby union, scuba diving, yoga, billy-cart racing, shooting, windsurfing, and kayaking; this group also included the use of playground equipment. Deaths that occurred in the "other" sports were: two from bushwalking, two from surfing, one from kayaking, one from windsurfing, and one from a shooting sport.

\section{Injury rates}

Table 2 shows the crude and age adjusted rates of serious sport/recreation injury and death. Overall, the injury and death rates are low, although the injury rates for men are 12 times higher than for women (table 2). Assuming similar participation rates and age distributions across Australia, it is estimated that about 295 people over the age of 14 years 
would be seriously injured and 94 would die each year Australia wide during sport/recreation.

Table 1 shows the rate of injury related to participation in those sports with available participation data. The highest participation adjusted rates of serious injury were associated with motor, power boat, and equestrian sports (table 1). Whereas Australian football ranked third with respect to frequency of serious injury, after adjustment for participation, it was ranked sixth. Injury rates could not be calculated for aero sports because of the lack of published participation data, and cycling participation figures did not differentiate $\mathrm{BMX}$ riding and mountain biking from general pedal cycling, precluding calculation of appropriate injury rates.

\section{Profile of cases of serious injury and death sustained} in sport/recreation that reached hospital

Injury data were available for all cases captured by the VSTR $(\mathrm{n}=158)$, and a brief summary is presented here. The median (range) ISS and hospital stay were 17 (1-54) and 8.1 (0.1-88.7) days respectively. A stay in the intensive care unit was required for $38 \%$ of patients, and $39 \%$ were unable to be discharged directly home.

The 158 patients sustained 611 injuries, an average of 3.9 (range 1-20) injuries per person. The head and spine were the most commonly injured body sites, accounting for $23 \%$ and $21 \%$ of all injuries respectively (table 3 ). Over $40 \%$ of all injuries were fractures $(44 \%)$, and intracranial $(16 \%)$ and internal organ $(13 \%)$ injuries were also common. Sixteen patients $(10 \%)$ suffered a spinal cord injury, and a third sustained a head injury classified as serious to critical $(\geqslant 3)$ according to the AIS.

The profile of injuries differed across the most common sports. The median number of injuries sustained by aero sport participants was six; $50 \%$ of the injuries were to the spine. Multiple injuries were also common in motorbike riders (median $=4$ ), with the injuries spread widely across the body regions (15-18\% across the thorax, head, spine, and upper extremity). Horse riders sustained a median of three injuries per case, with the head the most commonly injured region $(36 \%)$ followed by the thorax and upper extremity, which accounted for $14 \%$ and $12 \%$ of injuries respectively. The median number of injuries sustained while power boating was three, with injuries most commonly of the spine $(19 \%)$, head (16\%), and lower extremity (16\%). Football players sustained fewer injuries (median of two injuries per player), with the spine and abdomen the most commonly injured body regions ( $28 \%$ of injuries each).

Of the 53 cases with a serious head injury, over a third (34\%) were motor sport related and $21 \%$ were related to participation in equestrian activities. The breakdown of

Table 3 Body regions injured in sport and recreation related serious injury cases in Victoria, Australia 1 July 2001 to 30 June 2003 (inclusive) ( $n=158$ )

\begin{tabular}{lll}
\hline Body region & No & $\begin{array}{l}\text { \% of all } \\
\text { injuries }\end{array}$ \\
\hline Head & 141 & 23.1 \\
Spine & 131 & 21.4 \\
Thorax & 79 & 12.9 \\
Face & 67 & 11.0 \\
Upper extremity & 66 & 10.8 \\
Lower extremity & 61 & 10.0 \\
Abdomen and pelvic contents & 55 & 9.0 \\
External & 10 & 1.6 \\
Neck & 1 & 0.2 \\
Total & 611 & 100.0 \\
\hline
\end{tabular}

sports resulting in spinal cord injury was: motorbike riding $(n=7)$, snow skiing $(n=3)$, Australian football $(n=2)$, swimming $(\mathrm{n}=2)$, yoga $(\mathrm{n}=1)$, and parachuting $(\mathrm{n}=1)$.

\section{DISCUSSION}

This study is one of the few to describe serious sport/ recreation injuries in a defined geographic community. Using the combined sources of coronial, trauma registry, and population participation data, this study has identified the sport/recreation activities most commonly associated with serious injury and estimated the incidence of serious injury across these activities for the population of Victoria, Australia. Most of the injured were young ( $<45$ years), corresponding to the age group with the highest participation rates. ${ }^{17}$ Despite similar participation rates for both sexes, most of the injured were male $(81 \%)$. This finding is consistent with previous hospital admissions data ${ }^{6}$ and reflects the nature of male sport/recreation activities, as well as the potential for increased risk taking behaviour by men. ${ }^{19}{ }^{20}$ The findings identify motor, power boat, and equestrian sports as having the highest rates of serious injury.

Systematic monitoring of serious sport/recreation injury cases is uncommon. In the United States, the National Centre for Catastrophic Sports Injury Research (NCCSIR) has been operating since 1982. It uses a variety of methods, including the assistance of people working with athletes, a national newspaper clipping service, and associates of the researchers, to identify and capture information about eligible cases. ${ }^{7} 810$ However, although the NCCSIR does monitor severe sport related injuries across the United States, only school and collegiate sports are included. Unfortunately, there is no systematic approach to case identification. Cases can be missed or not identified until some time later, even years after their occurrence, and the accuracy of the information collected may be subjected to bias. ${ }^{8}{ }^{10}$ Participation data are not collected, and no measures of injury risk can be calculated. Although the NCCISR has been useful in the United States, the sports covered are largely irrelevant to the Australian population and do not reflect the pattern of its sport and recreation participation.

Active participation is not restricted to organised sport. Any system that captures only organised sports cases provides a limited picture of the epidemiology of serious sport/recreation injury. Until recently, most information about the more severe end of the sport/recreation injury spectrum has come from emergency department presentations and hospital admissions. ${ }^{56}$ However, information from these sources has limitations such as difficulties in identifying individual sport and recreation activities through the ICD systems ${ }^{56}$ and issues with defining participation numbers relevant to the captured population for the calculation of injury rates.

The population based nature of the VSTR and the NCIS, combined with participation figures, has provided a rare opportunity to estimate and rank serious sport/recreation injury rates. This study has estimated the rate of serious sport/recreation injury and death to be low at 1.8 and 0.6 cases per 100000 participants per year respectively compared with the estimated rate of hospital admission in Victoria for sport/recreation of 160/100 000 cases per year. ${ }^{6}$ The death rate from road traffic accidents in Victoria is 7 per 100000 people per year, in contrast with the relatively low rate of serious injury and death in sport/recreation. Nevertheless, multiple injuries were common, and injury rates adjusted for participation numbers suggest an excess risk of serious injury for participants in motor, power boat, and equestrian sports.

Drowning was the most common cause of death for sport/ recreation participants, with deaths occurring in a variety of water based sports such as fishing, power boating, swimming, kayaking, windsurfing, and surfing. Almost all 


\section{What is already known on this topic}

Limited information is available on serious injury and death resulting from sport and recreation. This information is necessary to highlight sports with excess risk of serious injury and to set priorities for injury prevention efforts.

non-drowning related deaths were the result of participation in motor and aero sports.

The definition of serious injury for this study was that used by the VSTR. This definition includes all injury related deaths and those with injuries severe enough to require intensive care or urgent surgery or to have an ISS $>15$. Although these inclusion criteria do not consistently capture near-drowning cases, the types of injury captured would be consistent with the NCCISR, which categorises injuries as fatal, non-fatal but resulting in permanent and severe functional disability, and serious injury without permanent functional disability. Our study was not able to make the distinction between serious injury with or without functional disability. How the NCCISR makes the delineation between these two groups is unclear as, in most cases, case information is collected as close as possible to the time of injury, ${ }^{10}$ and long term and routine follow up of cases does not appear to be undertaken. The AIS codes classify severity on a "threat to life" scale rather than the potential for long term disability. Whereas complete and incomplete spinal cord injuries will result in a neurological deficit and permanent disability, the predicted long term consequences of specific head and spinal injuries is not as clear. Studies investigating the long term outcomes of sport/ recreation injuries are necessary to fully understand the long term costs of these injuries.

The strengths of this study lie in the population based nature of the VSTR and the NCIS and the detailed information captured by these systems. Nevertheless, a number of limitations must be acknowledged, including the quality of the participation data used to calculate the injury rates. Our population participation data were taken from the ERASS, an annual survey of a small $(\mathrm{n}=13000)$ sample of the Australian population. ${ }^{17}$ Only limited information about the frequency of participation in the previous year is collected and not all sports are included in the report. As the participation data are collected from only a small sample of the Australian population, there is the potential for bias and sampling error, which may impact on the injury rates calculated. In addition, the small number of serious injury cases for some sports precluded the calculation of reliable injury rates, preventing a detailed comparison of all sport/ recreation activities.

The extrapolation of the Victorian figures to the rest of Australia provides an estimate only of the expected number of cases of serious injury and death per year, and assumes similar participation rates across Australia. According to figures from the Australian Bureau of Statistics, the rate of participation in Victoria is similar to the rest of Australia for most sports. ${ }^{13}$ However, there are variations in participation in sports, such as the football codes, across the states of Australia.

Identification of sport/recreation cases relies on the detailed text narrative in the VSTR. The sport or recreational activity participated in is consistently collected, but very fine detail about the injury event such as use of protective equipment, details of equipment failure, and other contributing factors is not always documented. Nevertheless, the key aims of a registry are to monitor patterns of injury and identify potential problem areas, and the VSTR has met these
What this study adds

This population based study found that the rate of serious injury and death in Victoria, Australia was low. Injury rates adjusted for participation numbers suggest an excess risk of serious injury for participants in motor, power boat, and equestrian sports. Drowning was the predominant cause of death in sport and recreation, and participation in motor and aero sports was the most common cause of non-drowning deaths.

aims through the identification of sport and recreational activities associated with serious injury and the pattern of injuries sustained.

\section{CONCLUSION}

Overall, the rate of serious injury related to sport/recreation participation in Victoria is 1.8 cases of serious injury, and 0.6 deaths, per 100000 participants per year. It is estimated that there are 295 serious injury cases and 94 deaths each year related to sport/recreation in Australia. The rate of injury was highest for motor, power boat, and equestrian sport, but serious injuries were also common in aero sports and Australian football. The findings of this study identify these sports as priorities for further research into injury prevention strategies. Drowning was the most common cause of death for sport and recreation participants, highlighting this for prevention efforts.

\section{ACKNOWLEDGEMENTS}

The Victorian State Trauma Registry (VSTORM) is funded by a grant from the Victorian Trauma Foundation and assistance from the Department of Human Services, Victoria. We acknowledge the help provided by VSTORM staff and members of the steering committee. In particular, Andrew Hannaford is sincerely thanked for his assistance. Data from the NCIS were provided by the Monash University National Centre for Coronial Information. BJG and CFF were supported by a Public Health Fellowship (237024) from the National Health and Medical Research Council (NHMRC) and an NHMRC Principal Research Fellowship respectively.

\section{Authors' affiliations}

B J Gabbe, P A Cameron, O D Williamson, Monash University, Melbourne, Australia

C F Finch, University of New South Wales, Sydney, NSW, Australia

Competing interests: none declared

\section{REFERENCES}

1 Medibank Private. Medibank Private sports injuries report. Melbourne: Medibank Private Limited, 2004.

2 Finch C, Owen N, Price R. Current injury or disability as a barrier to being more physically active. Med Sci Sport Exerc 2001;33:778-82.

3 Kelly K, Lissel H, Rowe B, et al. Sport and recreation-related head injuries treated in the emergency department. Clin J Sport Med 2001;11:77-81.

4 Gotsch K, Annest J, Holmgreen P, et al. Nonfatal sports- and recreationrelated injuries treated in emergency departments, United States, July 2000June 2001. JAMA 2002;288:1977-8.

5 Finch C, Valuri G, Ozanne-Smith J. Sport and active recreation injuries in Australia: evidence from emergency department presentations. $\mathrm{Br} J$ Sports Med 1998;32:220-5.

6 Cassell E, Finch C, Stathakis V. Epidemiology of medically treated sport and active recreation injuries in the Latrobe Valley, Victoria, Australia. Br J Sports Med 2003;37:405-9.

7 Boden B, Pasquina P, Johnson J, et al. Catastrophic injuries in pole-vaulters. Am J Sports Med 2001;29:50-4.

8 Boden B, Tacchetti R, Mueller F. Catastrophic cheerleading injuries. Am J Sports Med 2003;31:881-8.

9 Luckstead E, Patel D. Catastrophic pediatric sports injuries. Pediatr Clin North Am 2002;49:581-91.

10 Mueller F. Catastrophic head injuries in high school and collegiate sports J Athl Train 2001;36:312-15. 
11 Conn J, Annest J, Gilchrist J. Sports and recreation related iniury episodes in the US population, 1997-1999. Inj Prev 2003;9:117-23.

12 Mummery W, Spence J, Vincenten J, et al. A descriptive epidemiology of sport and recreation injuries in a population-based sample: results from the Alberta Sport and Recreation Injury Survey (ASRIS). Can J Public Health 1998;89:53-6.

13 Australian Bureau of Statistics. Participation in sport and physical activities Canberra: Australian Bureau of Statistics, 2002, Report No 4177.0.

14 Cameron P, Finch C, Gabbe B, et al. Developing Australia's first statewide trauma registry: What are the lessons? Aust N Z J Surg 2004;74:424-8.

15 Committee on Medical Aspects of Automotive Safety. Rating the severity of tissue damage: the abbreviated scale. JAMA 1971;215:277-80.
16 Senkowski C, McKenney M. Trauma scoring systems: a review. J Am Coll Surg 1999; 189:491-503.

17 Standing Committee on Recreation and Sport. Participation in exercise, recreation and sport. Canberra: State/Territory Departments of Sport and Recreation, 2002

18 Australian Bureau of Statistics. The Australian Census of Population and Housing: Expanded Community Profile-Victoria and Australia. Canberra: Australian Bureau of Statistics, 2001, Catalogue No 2005.0.

19 Kontos A. Perceived risk, risk taking, estimation of ability and injury among adolescent sport participants. J Pediatr Psychol 2004:29:447-55.

20 Green M, Turner C, Purdie D, et al. Injury related risk behaviour: a study of Australian skydivers. J Sci Med Sport 2003;6:166-75.

\section{$\mathrm{ECHO}$}

\section{Tai chi seems ineffective for rheumatoid arthritis}

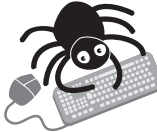

Please visit the British Journal of Sports Medicine website [www. bjsportmed. com] for a link to the full text of this article.
Deumatoid arthritis, unlike osteoarthritis, does not improve with tai chi exercise, initial results have suggested, but further investigation is warranted, the researchers say, because of shortcomings of their pilot.

The study took 15 women aged 40-70 (mean 57.0 (SD 8.6)) years with moderate disability one year before the study at random from the rheumatoid arthritis register in Oslo. Norway, and followed them over eight weeks of a tai chi programme modified for arthritis sufferers. This comprised two 45 minute sessions a week and was given by trained tai chi instructors. Various objective and self reported measures of disease, performance, and health were recorded at baseline, four weeks into the programme, and one week after the programme was completed.

Hoped for improvements, as have been seen in osteoarthritis, did not materialise. Self reported measures and performance, such as muscle strength, joint flexibility, and balance, and cardiovascular fitness remained the same, as did biochemical measures of inflammation and disease activity. Nevertheless, all bar two women enjoyed the programme much more than their usual physiotherapy and found it more useful.

However, these initial results should not deter further exploratory research with more patients; a longer programme of 10-12 weeks, as generally recommended; and more responsive chosen measures, the researchers maintain.

\Uhlig T, et al. Annals of the Rheumatic Diseases 2005;64:507-509. 